\title{
DEVELOPMENT AND IMPROVEMENT IN THE SNAKE ANTIVENOM PRODUCTION
}

THESIS: V. Morais submitted this dissertation for his Master in Biotechnology at Science Faculty, University of the Republic, UDELAR, Montevideo, Uruguay, 2005.

Advisor: Professor Hugo A. Massaldi

ABSTRACT: Antivenoms constitute the only effective treatment for snakebite envenomation. However, heterologous sera have the potential to provoke adverse reactions in patients, some of them being very dangerous like anaphylactic shock. Antivenoms development aims to obtain a pure product, with a minor degree of adverse reactions and accessible costs. In this work, we propose to investigate about the following points: 1 - pepsin digestion of immunoglobulins to obtain $F\left(a b^{\prime}\right)_{2}$ fragment; 2 - immunoglobulin purification based on caprylic acid; 3 - economical analysis of the overall process. We could determinate the best conditions to the overall immunoglobulin digestion to $\mathrm{F}(\mathrm{ab})_{2}$. If the digestion is made using plasma, the best conditions are: $\mathrm{pH} 3.0,2$ hours of incubation, and $2 \mathrm{~g} / \mathrm{l}$ of pepsin. If the digestion is carried with purified immunoglobulins, the digestion could be run under the same conditions but at $\mathrm{pH}$ 3.2. In both cases, digestion at upper $\mathrm{pH}$ is possible but it is necessary to increase the enzyme concentration and the reaction time. In addition, the activity lost due to the pepsin digestion was quantified; the most favorable condition is about $60 \%$ of activity yielded. The optimal parameters for immunoglobulin purification by caprylic acid were evaluated. This acid precipitates almost all plasma proteins, except immunoglobulins. This method shows the best production-cost relationship. It is possible to obtain purity and yields above $90 \%$. At last, we present an economical analysis of all the factors affecting the production costs. We concluded that for snake antivenom production, the large contribution of fixed costs to the total cost makes necessary, or convenient, to try to increase the number of vials (doses) produced per year using the same facility and technology. Since many producing countries only manufacture the necessary number of doses to cover the country requirements, their systems of production are underutilized during more or less extensive periods, which causes an augment of the unitary cost. The fixed costs are represented by the amortization of the installations and equipment (calculated as: cost of the equipment or installation/useful life) and particularly by the permanent work force. Furthermore, it is very important to obtain a good immunization titer and use an efficient method to process the plasma in order to improve economical yields.

KEY WORDS: antivenom, purification process, pepsin, caprylic acid, economical evaluation.

CORRESPONDENCE TO:

VICTOR MORAIS, Departamento de Desarrollo Biotecnológico y Producción, Instituto de Higiene, Facultad de Medicina, UDELAR, Av A. Navarro 3051, 11600, Montevideo, Uruguay. Email: vmorais@higiene.edu.uy. 